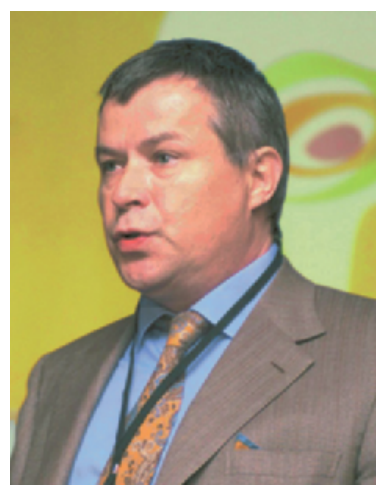

—J.P. Elsig

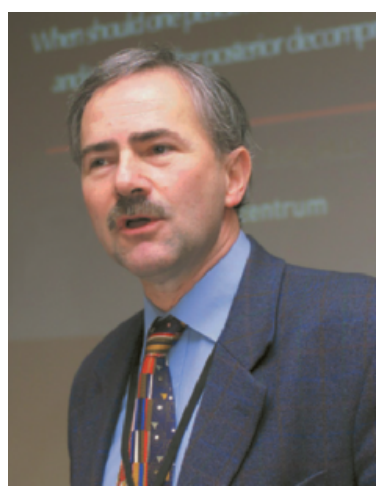

-D.L. Kaech

\section{Intraoperative imaging for improved safety and quality control in spine surgery}

This ASN\&J issue focuses on intraoperative imaging. It seems indeed a relevant and topical subject to address. Every spine surgeon is familiar with the basics of intraoperative fluoroscopy to check the level of a surgery, the position of various implants - a routinely performed imaging investigation - as daily quality control.

By adding contrast medium, more insight into the anatomy of the spinal canal is possible, as with intraoperative myelography, or with epidurography, by applying this contrast medium, e.g. after an anterior discectomy, including the position check during insertion of radiolucent implants (such as cages for instance).

This issue opens with an interview with Prof. Dosch, dedicated to the historical evolution of intraoperative imaging.

According to the modern needs for maximal safety, further technological advancements were made during the last decade. Computer assisted systems were developed to allow for navigation, notably minimizing the risk of screw misplacement, but also improving the less invasive but more precise approach e.g., for tumour resection. We publish a paper dealing with navigation by Prof. Schönmayr et al. and another one by J.-C. Le Huec et al. introducing the O-arm ${ }^{\circledR}$, which provides 3-D viewing of the spine during a procedure, like a CT-scan.

However, the costs involved are always a matter of concern, as navigation and O-arm ${ }^{\circledR}$ equipments are expensive.

To improve safety during pedicular screw placement, the PediGuard ${ }^{\circledR}$ was developed. It is a pedicle finder and probe with a sensor at the tip, which gives an acoustic signal depending on the quality of bone. A change in the "bleep signal" indicates that the surgeon is no longer in the cancellous bone, thus giving an early warning when, for example, the cortical bone is violated and the planned screw is likely to injure the nerve root.

Although not fully exhaustive on the latest trends in intraoperative imaging in late 2010, this overview reflects current options for optimising accuracy and safety in spine surgery. •

J.P. Elsig and D.L. Kaech 